\title{
Cross-sector surveys assessing perceptions of key stakeholders towards barriers, concerns and facilitators to the appropriate use of adaptive designs in confirmatory trials
}

Munyaradzi Dimairo ${ }^{{ }^{*}}$, Steven A. Julious ${ }^{1}$, Susan Todd ${ }^{2}$, Jonathan P. Nicholl ${ }^{1}$ and Jonathan Boote ${ }^{1,3}$

\begin{abstract}
Background: Appropriately conducted adaptive designs (ADs) offer many potential advantages over conventional trials. They make better use of accruing data, potentially saving time, trial participants, and limited resources compared to conventional, fixed sample size designs. However, one can argue that ADs are not implemented as often as they should be, particularly in publicly funded confirmatory trials. This study explored barriers, concerns, and potential facilitators to the appropriate use of ADs in confirmatory trials among key stakeholders.

Methods: We conducted three cross-sectional, online parallel surveys between November 2014 and January 2015. The surveys were based upon findings drawn from in-depth interviews of key research stakeholders, predominantly in the UK, and targeted Clinical Trials Units (CTUs), public funders, and private sector organisations. Response rates were as follows: 30(55 \%) UK CTUs, $17(68 \%)$ private sector, and 86(41 \%) public funders. A Rating Scale Model was used to rank barriers and concerns in order of perceived importance for prioritisation.

Results: Top-ranked barriers included the lack of bridge funding accessible to UK CTUs to support the design of ADs, limited practical implementation knowledge, preference for traditional mainstream designs, difficulties in marketing ADs to key stakeholders, time constraints to support ADs relative to competing priorities, lack of applied training, and insufficient access to case studies of undertaken ADs to facilitate practical learning and successful implementation. Associated practical complexities and inadequate data management infrastructure to support ADs were reported as more pronounced in the private sector. For funders of public research, the inadequate description of the rationale, scope, and decision-making criteria to guide the planned AD in grant proposals by researchers were all viewed as major obstacles.
\end{abstract}

Conclusions: There are still persistent and important perceptions of individual and organisational obstacles hampering the use of ADs in confirmatory trials research. Stakeholder perceptions about barriers are largely consistent across sectors, with a few exceptions that reflect differences in organisations' funding structures, experiences and characterisation of study interventions. Most barriers appear connected to a lack of practical implementation knowledge and applied training, and limited access to case studies to facilitate practical learning.

Keywords: Adaptive designs, flexible designs, barriers, surveys, confirmatory trials, Phase 3, clinical trials, early stopping, interim analyses

\footnotetext{
* Correspondence: m.dimairo@sheffield.ac.uk

'ScHARR, Regent Court, University of Sheffield, 30 Regent Street, S1 4DA,

Sheffield, UK

Full list of author information is available at the end of the article
} 


\section{Background}

"... there can be no objection to the use of data, however meagre, as a guide to action required before more can be collected ...." Thompson, 1933 [1]

The role of clinical trial adaptation has started to come to the fore in recent years, with numerous methodological developments over the past 40 years aimed at improving efficiency in the design and conduct of clinical trials $[2,3]$. Researchers and policymakers are now considering adaptive designs (ADs) more often than before, and the use of ADs is increasing [4-6]. However, one could still argue that ADs, particularly in confirmatory trials, remain underutilised.

Recent research has investigated barriers and opportunities to the use of ADs, with emphasis on the pharmaceutical trial perspective [4, 6-8], the US setting $[9,10]$, and publicly funded early phase trials in the UK [11]. Some of these previous studies have been informal in nature, and some important barriers to successful implementation could potentially have been overlooked. Furthermore, the attitudes of public funders towards the use of ADs have not been formally explored, and little focus has been directed to confirmatory trials in the UK setting. To address these gaps in previous research, we undertook multi-disciplinary and cross-sector in-depth interviews of key stakeholders in clinical trials research to inform the design of subsequent surveys [12]. Interviews were undertaken with a range of researchers including public funding panel chairs and members, statisticians, leaders of UK Clinical Trials Units (CTUs), chief investigators, regulators, health economists, and data monitoring committee members.

Based upon themes generated from the interview findings [12], we conducted quantitative surveys aimed at public and private sector researchers, and public funders, predominantly in the UK. The main objectives of the surveys were as follows:

1. Investigate perceived barriers to and concerns regarding the use of ADs in confirmatory trials and gauge the opinions of key stakeholders, such as public funders;

2. Rank the barriers and concerns in order of perceived importance for prioritisation and compare and contrast these rankings between public and private sector researchers;

3. Investigate the types of ADs being implemented in practice in confirmatory trials; and

4. With a focus on publicly funded trials, explore ways to address perceived barriers and concerns and propose recommendations in cases where ADs may potentially offer added benefits to standard trial designs.

\section{Methods}

Between November 2014 and January 2015, we conducted three cross-sectional, parallel, quantitative online surveys tailored for UK CTUs, private sector organisations, and public funders, exploring perceptions towards and potential facilitators to the appropriate use of ADs in confirmatory trials.

\section{Surveys' sampling frames}

This research paid attention to researchers both in the private and public sector as a collaborative platform for addressing cross-sector roadblocks to the appropriate use of ADs. In addition, the conduct of clinical trials research and approval of effective and safe healthcare interventions into medical practice is a complex process involving various key stakeholders, such as funders/ sponsors, researchers and policymakers. Hence, prospective participants were identified as follows:

The United Kingdom Clinical Research Collaboration (UK CRC) comprises a network of registered CTUs with expertise to coordinate and support high quality conduct of clinical trials [13]. Consequently, major UK public funders such as the National Institute for Health Research (NIHR) and the Medical Research Council (MRC) require, as part of their funding policy, the involvement of UK CRC-registered CTUs with the vision to improve quality in the conduct and delivery of publicly funded clinical trials. Overall, there were 55 registered CTUs (2013/2014) across the UK [13], which we contacted to take part in our research.

The NIHR is one of the major UK public funders of medical research, contributing about a third of total research funding - spending over $£ 1$ billion in $2013 / 2014$ [14]. Within the NIHR programme, the Health Technology Assessment (HTA) is the largest funding stream for the commissioning of independent research, which includes predominantly confirmatory trials to assess clinical and cost effectiveness, and the broader impact of healthcare interventions that have been tailored for the National Health Service (NHS) [15]. The HTA programme has four boards supported by five advisory panels and a priority group, some of whose members are publicly contactable [16]. There are other NIHR funding streams supporting a smaller proportion of confirmatory trials, such as the Efficacy Mechanism and Evaluation (EME), and Research for Patient Benefit (RfPB). In acknowledging the crucial role of charity funders in clinical trials research and to capture diverse views, Cancer Research UK (CR UK) advisory panel members were also contacted because it is one of the largest UK charities funding confirmatory trials. In addition, huge opportunities to use adaptive designs are perceived in therapeutic areas such as oncology [12]. We also approached a second large UK charity funder, but 
unfortunately, the coordinating team was uncomfortable about us contacting their boards and panel members. Overall, 212 contactable public funding board members and advisory panel members from HTA $(n=110)$, EME $(n=20)$, RfPB $(n=40)$, and CR UK $(n=42)$ were approached to take part.

Private sector trials research is mostly conducted by pharmaceutical and biotech companies, and Contract Research Organisations (CROs). Twenty-five companies with which we had direct contact were approached to take part: pharmaceutical or biotech organisations $(n=13)$ and CROs $(n=12)$.

\section{Design of survey instruments}

A list of themes on perceived barriers, concerns, and potential facilitators was compiled based on prior findings from our in-depth interview study [12]. These themes were then grouped to develop survey instruments depending on to whom they pertained: public funders, UK CTUs, and private sector. Most of the questions were of a closed form; however, we included open-ended questions for respondents to add detailed responses where applicable. We accessed the perceptions of respondents on the importance of barriers and concerns towards, and usefulness of, potential facilitators to the appropriate use of ADs in confirmatory trials using widely accepted Likert Scales [17]. Most questions for the UK CTU and private sector surveys were phrased consistently, except on those occasions when specific questions were unique to a certain sector. Instruments were internally reviewed and piloted prior to the launch of the online surveys. Figure 1 displays a snapshot of CTUspecific questions on barriers.

\section{Approaching targeted participants}

The Directors or designated Senior Statisticians were contacted to complete the online survey instrument tailored for UK CTUs. Only one response per CTU representative was permitted. Two rounds of email invitations with an information sheet were sent through the UK CRC-registered CTU network of Directors and Senior Statistician representatives. A third round of personalised emails were directly sent to some non-responders $(\mathrm{n}=21)$ of the previous two rounds.

Public funding boards and panels chairs and ordinary and lay members were approached through email to complete an online survey tailored for public funders. One round of invitation emails was sent to panel chairs and vice chairs, and some panel members through the NIHR programme coordinators. Some members were directly contacted by personalised emails using online, publicly accessible contact details.

For the private sector; two rounds of emails were directly sent to trial Research Leaders or designated Principal or Senior Statisticians from 25 organisations with whom we had contact. Multiple responses from the organisations with several trial research groups were permitted where applicable.

All cross-sector participants were given between 3 and 8 weeks to complete the online surveys.

\section{Statistical analysis and reporting}

Descriptive statistics were generated, aided by forest plots and clustered bar charts to display respondent perceptions. A Rating Scale Model for ordered response items [18] was employed to rank the perceived

Q8. To what extent do you view the following as main barriers to the use of ADs when appropriate in confirmatory trials within your CTU?

Lack of awareness of benefits of ADs
Lack of awareness of when ADs are appropriate
Lack of awareness of which scope of ADs are acceptable
in confirmatory trials
Difficulties in marketing ADs to key stakeholders in trials
research (such as collaborators, funders and regulators)
Research team being more comfortable with the
conventional mainstream designs compared to ADs
Lack of practical implementation knowledge
Lack of applied training to facilitate practical
implementation
Fig. 1 A snapshot of the UK CTUs survey instrument


importance of barriers and concerns as characterised by the 'difficulty' parameter using the RUMM2030 software [19]. A respondent's log odds of choosing a higher category of an item on an importance (or concern) scale over the previous adjacent category was modelled as a function of the ability of the respondents, perceived level of importance (or concern) attached to an item, and threshold parameters of items as follows [18]:

$$
\ln \left(\frac{p_{n i k}}{p_{n i(k-1)}}\right)=\theta_{n}-\left(\delta_{i}+\tau_{k}\right),
$$

where $p_{n i k}$ is the probability of a respondent $n$ with ability $\theta_{n}$ choosing category $k$ for an item $i ; k=0,1,2, \ldots ; m$ represents the ordered choices, and $m$ is the number of item steps; $\delta_{i}$ is the 'difficulty' of item $i$, which is the importance location parameter of interest; $\tau_{k}$ is the threshold parameter corresponding to choice $k$ in item $i$; and $\theta_{n}$ and $\tau_{k}$ are nuisance parameters.

The research utilised a recent review of existing guidance on the reporting of survey research and attempted to use relevant items to enhance its reporting [20]. To facilitate interpretation and consistency, barriers and concerns to the use of ADs are presented in order of perceived importance. Proportions of item responses, estimates of the importance parameters with associated $95 \%$ confidence intervals (CIs), and item rank are presented.

\section{Ethical approval}

This study received favourable ethical approval (0676) from the School of Health and Related Research (ScHARR) Ethics Committee at the University of Sheffield. Participants were only permitted to complete the survey after consenting, which was the opening question on all survey instruments.

\section{Results}

\section{Response rates}

We observed a UK CTU crude response rate of $55 \%$ (30/55). In addition, $46 \%(25 / 55)$ of respondents completed all key questions (barriers, concerns, and potential facilitators). Approximately $68 \%$ (17/25) of the organisations responded to the private sector survey. Of these, $52 \%(13 / 25)$ responded to all key survey questions. Approximately $41 \%(86 / 212)$ of the respondents completed the online survey tailored for public funders. However, the response rate to all key questions was around $30 \%$ (64/212). Non-response feedback from two UK CTUs and six public funders cited a lack of basic understanding of ADs to meaningfully contribute to the survey as the main reason for non-participation.

\section{Baseline characteristics of respondents}

Of the 30 UK CTUs respondents, 10(33\%) and 18(60\%) were Directors and Senior Statisticians respectively, and two did not state their role. CTUs covered a wide geographical area across the UK and represented many therapeutic areas of clinical trials research. Overlapping and diverse therapeutic areas of research include oncology $(\mathrm{n}=13)$, mental health $(\mathrm{n}=11)$, primary care $(\mathrm{n}=9)$, public health $(n=9)$, musculoskeletal $(n=8)$, respiratory $(n=8)$, cardiovascular $(n=7)$, diabetes $(n=7)$, health services $(n=7)$, emergency medicine $(n=6)$, infectious $(n=2)$, rare or orphan diseases $(n=2)$, perinatal medicine $(\mathrm{n}=1)$, surgical interventions $(\mathrm{n}=1)$, and other $(\mathrm{n}=3)$. Figure 2 displays the approximate distribution of the study interventions as a percentage of the total number of trials based on complete data reported. In addition, the distribution of the trials requiring regulatory approval (such as from the MHRA, EMA, or FDA) beyond standard ethics had a median (IQR) of $50 \%$ (16\% to $80 \%$ ), based on 23 complete responses.

The expertise of public funder respondents in clinical trials research was diverse and overlapping, representing the composition of public funding boards and advisory panels. These included trial statisticians 11(13\%), chief investigators $40(47 \%)$, trial methodologists $20(23 \%)$, trial management experts 6(7\%), clinical experts 23(27\%), health economists $9(10 \%)$, Independent Data Monitoring Committee 33(38 \%) and Trial Steering Committee members 24(28\%), CTU directors $12(14 \%)$, patient representatives $7(8 \%)$, and other $6(7 \%)$. Ordinary members and Chairs or Vice Chairs of boards or panels constituted 63(73\%) and 10(12\%), respectively. Most members $(65 \%)$ had served for less than 5 years on their current funding boards or panels. Of the 17 industry respondents, 9(56\%) and 7(44\%) were from pharmaceutical or biotech organisations and CROs, respectively, and one did not state the nature of their organisation. Private sector respondents were predominantly Lead Statisticians in the UK 13(81 \%).

\section{UK CTU perceptions of barriers to ADs use in confirmatory trials}

The distribution of respondents' opinions and rank of barriers, based on estimates of the perceived relative importance parameters with associated $95 \%$ CIs, from the Rating Scale Model are displayed in Fig. 3. The more negative or smaller the relative importance parameter, the more important respondents perceived the barrier. Supplementary summary data with detailed description of survey questions relating to the barrier items presented in Fig. 3 are provided (see Additional file 1).

The lack of bridge funding accessible to CTUs to support the design work of complex and time-consuming ADs was perceived as the top-ranked obstacle impeding 


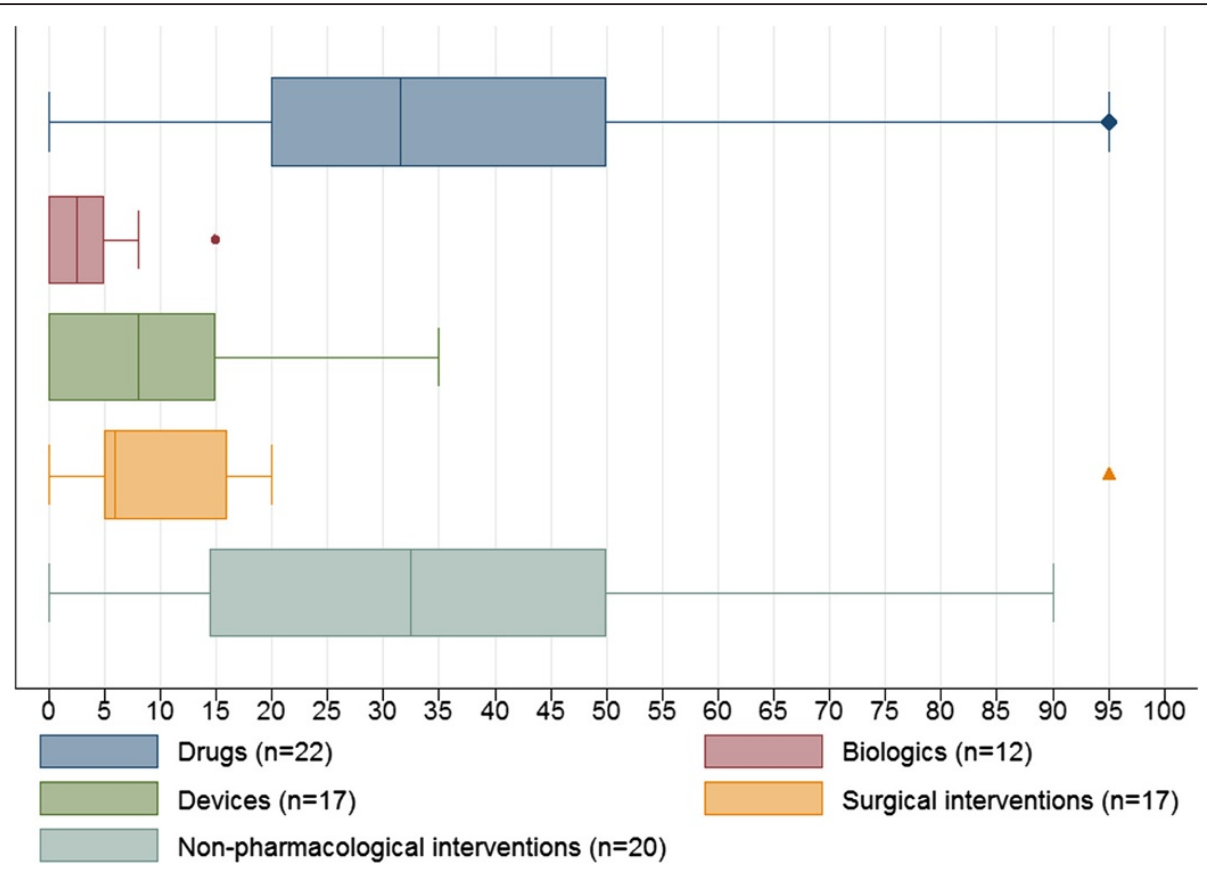

Fig. 2 The distribution of the nature of interventions investigated by UK Clinical Trials Unit (CTU) respondents

the routine use of Ads and was reported by $8(32 \%)$ and $12(48 \%)$ respondents as an 'extremely' or and 'at least moderately' important barrier, respectively. The lack of practical implementation knowledge and hands-on experience was the second highest ranked barrier, with
6(24\%) 'extremely' and 15(60\%) 'at least moderately' important rankings. The opinions of respondents suggested that research teams within the UK CTUs have a strong preference for traditional mainstream designs which they know well and feel uncomfortable supporting

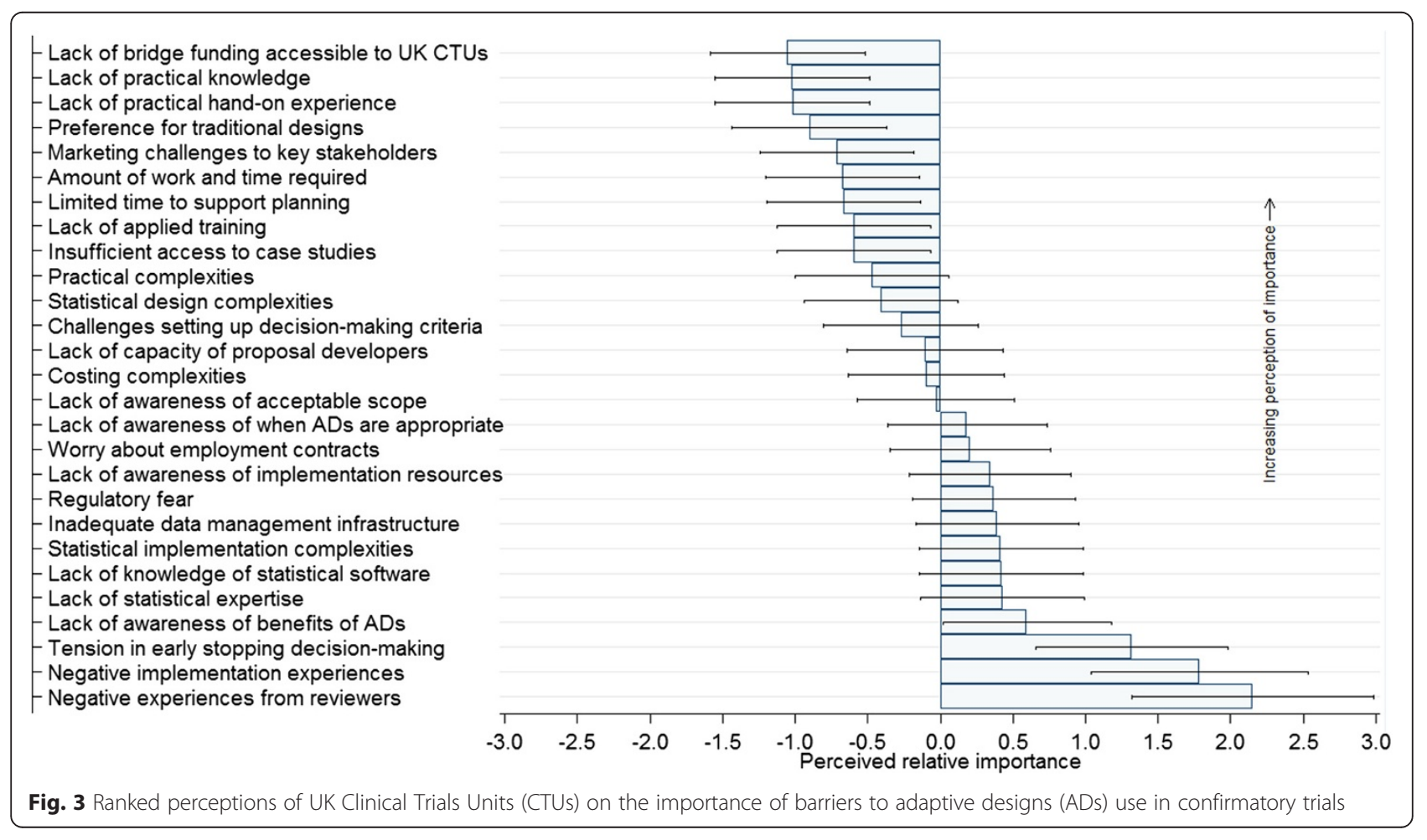


ADs even when they are appropriate. Only 3(12 \%) respondents did not view it as an important barrier, whereas $6(24 \%)$ and $12(48 \%)$ respondents reported it as an 'extremely' and 'at least moderately' important barrier, respectively (see Additional file 1 ).

Thirteen (52\%) respondents reported difficulties faced by trialists in marketing ADs to key stakeholders in trials research, such as clinical collaborators, funders, and regulators as 'at least a moderately' important barrier. The amount of time and effort required to support the design of ADs, and the time constraints relative to competing priorities of the traditional mainstream designs was reported as 'at least an important' barrier by $12(48 \%)$ respondents. The lack of applied training and insufficient access to case studies on ADs to facilitate practical learning and implementation were reported among the top-ranked barriers. Statistical complexities such as simulations work as part of design, practical implementation complexities and difficulties faced by clinical trialists in setting up acceptable planned decision-making criteria to guide the adaptation were among some of the middle-ranked barriers.

Barriers reported as 'not at all' important by many respondents were negative experiences based on funders' or reviewers' comments (76\%), negative implementation experiences (76\%), early stopping decision-making tensions among key decision-makers $(60 \%)$, and lack of awareness of benefits of ADs (48\%).
UK public funder perceptions on barriers to the use of ADs in confirmatory trials

Public funder respondents ranked the importance of most barriers considered with a small degree of differentiation (Fig. 4). Supplementary summary data with detailed description of survey questions relating to barrier items presented in Fig. 4 are provided (see Additional file 2). The preference of public funders for traditional mainstream designs over ADs and their risk-averse attitude to fund projects associated with a high degree of financial uncertainty were among the most important barriers reported. Researchers' inadequate description of the rationale for using an $\mathrm{AD}$ and decision criteria to guide the $\mathrm{AD}$ in grant proposals were reported as at least moderately' important barriers by $60 \%$ and $53 \%$ of respondents, respectively, thus making the review process challenging. The dearth of expert proposal reviewers to provide advisory support to funders during their grant commissioning process was selected by $32(55 \%)$ and $11(19 \%)$ of respondents, as a 'moderately' or 'extremely' important barrier, respectively. Inadequate descriptions by the researchers of the proposed ADs and the scope of these descriptions in the grant applications were also ranked among the top-ranked barriers. Some of the middle-ranked barriers were lack of commissioning experience of $\mathrm{AD}$-related research, and lack of awareness of acceptable scope of ADs and when they are appropriate in the confirmatory setting.
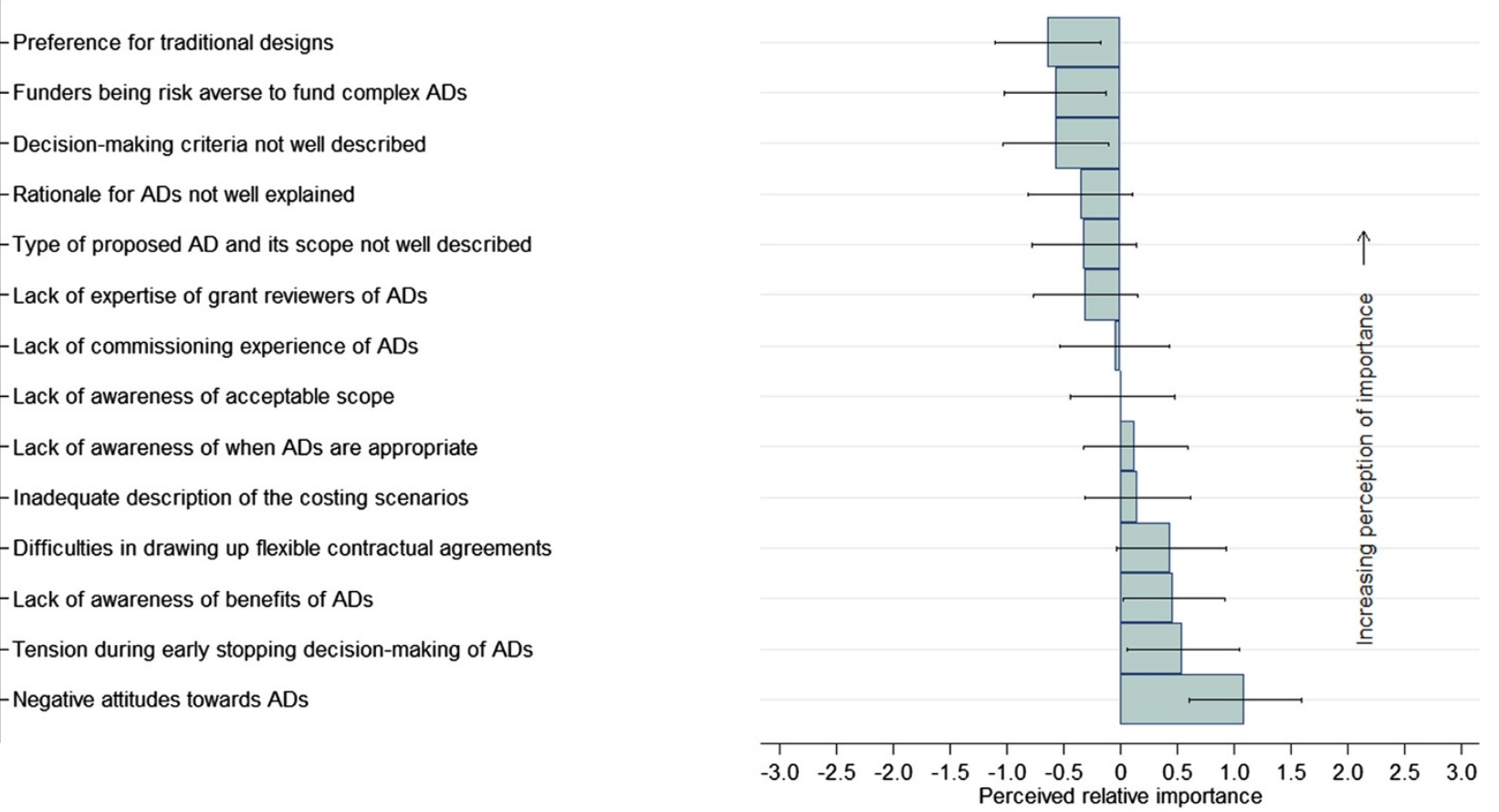

Fig. 4 Ranked perceptions of UK public funders on the importance of barriers to adaptive designs (ADs) use in confirmatory trials 
Challenges faced by funders in drawing up contractual agreements that are suitable to support ADs were reported by $38 \%$ and $10 \%$ of respondents to be a 'moderately' or 'extremely' important barrier. The lowest ranked of the perceived barriers were early trial stopping decision-making tensions among key decision-makers and negative attitudes towards ADs by some of the funding boards and panel members.

\section{Private sector perceptions of barriers to the use of ADs in confirmatory trials}

In general, the perceptions of UK CTUs and the private sector on the importance of different barriers are consistent; however, there are a few exceptions (Figs. 3 and 5). For instance, complexities during practical implementation, inadequate data management infrastructure, and fear of risking regulatory approval appear to be very prominent in the private sector (marked in red diamonds on Fig. 5). In contrast, the lack of bridge funding to support developmental design work and worry about research staff employment contracts when trials are stopped early were highly and middle rated in the public sector, respectively (marked in blue squares on Fig. 5). The top-ranked barriers reported to be 'at least moderately' important were as follows (see Additional file 3): the dearth of practical implementation knowledge 9(69\%); time constraints to support the planning of complex ADs relative to competing priorities of traditional mainstream designs 6(46\%); associated practical complexities during implementation of ADs 9(69\%); inadequate data management infrastructure for timely data capturing, cleaning, and processing to support ADs 5(42\%); the dearth of applied training to facilitate practical implementation $9(69 \%)$ and lack of hands-on practical experience $8(62 \%)$; limited access to case studies of the few ADs that have been implemented to facilitate practical learning 6(46\%); and research teams being more comfortable with traditional mainstream designs $8(62 \%)$.

The middle ranked important barriers reported with little degree of separation between them were the lack of awareness of acceptable scope of ADs in confirmatory trials; enormous amount of time and effort required during planning; fear of regulatory reluctance and risk of jeopardising chances of approval; difficulties faced in marketing $\mathrm{ADs}$ to key stakeholders; the lack of awareness of when ADs are appropriate; and difficulties faced in setting up upfront, acceptable decision-making criteria to guide trial adaptation (Fig. 5, Additional file 3).

Barriers reported as unimportant by a sizable number of respondents were a lack of awareness of implementation resources $6(46 \%)$, lack of knowledge of existing AD-related statistical software 6(46\%), lack of motivational support from Research and Development (R \& D) $6(46 \%)$, negative regulatory experiences $5(38 \%)$, lack of expertise to support planning 5(38\%), costing complexities during planning 6(46\%), negative experiences during

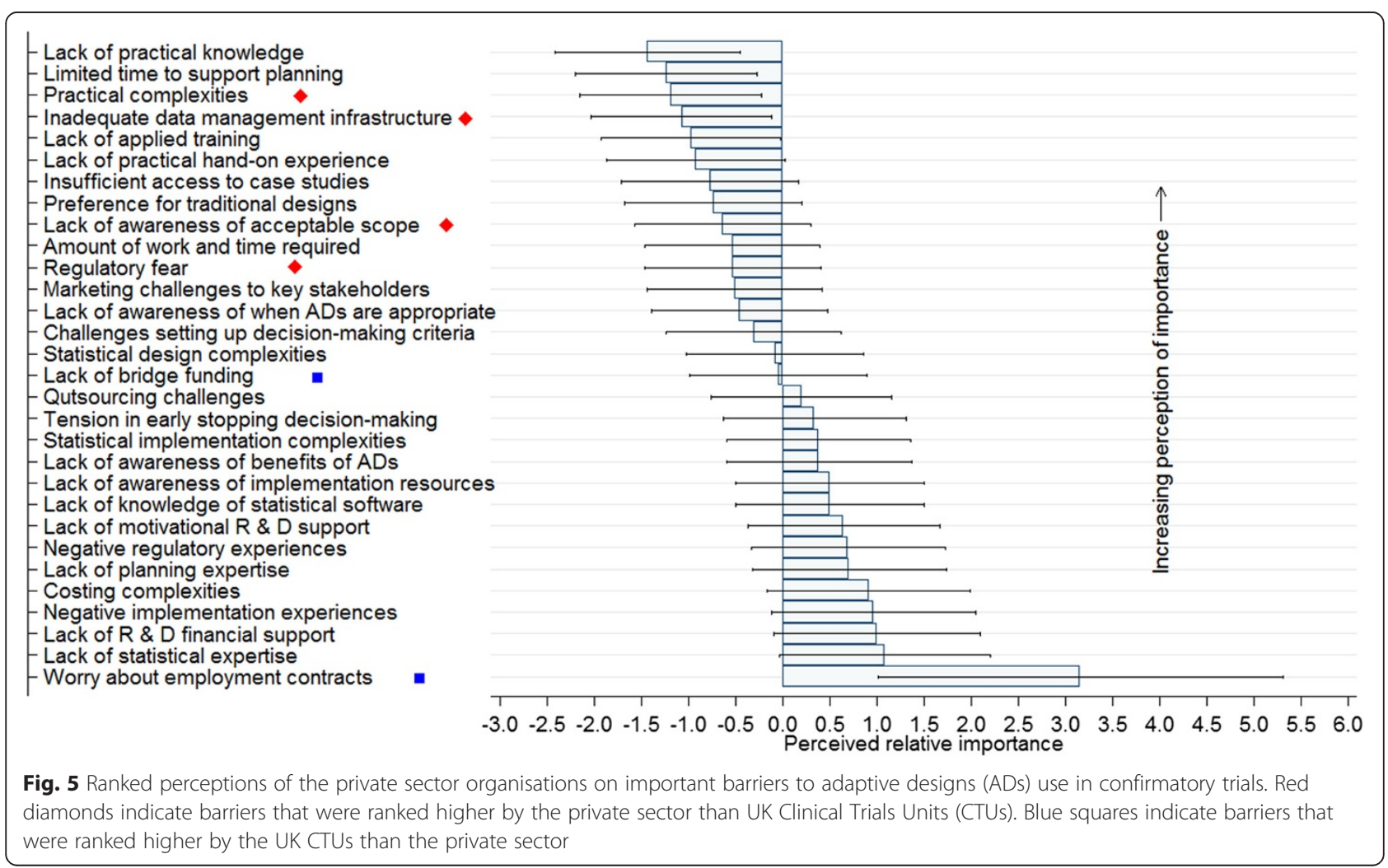


implementation 7(54\%), insufficient R \& D financial support to invest in $\mathrm{AD}$ infrastructure $8(62 \%)$, the dearth of statistical expertise to support ADs $8(62 \%)$, and worry about staff employment contracts when trials are stopped early $10(77 \%)$.

\section{Cross-sector perceived concerns about the use of ADs in confirmatory trials}

The early stopping of trials (or treatment arms) for futility - as soon as there is sufficient evidence of no benefit - was the least ranked concern across sectors (see Additional file 4). Concerns about the robustness of ADs in decision-making and the acceptability of findings to change medical practice when trials are stopped early appeared more pronounced among public funder respondents. The early stopping of trials for efficacy or non-inferiority were top-ranked concerns reported by UK CTUs and private sector respondents, respectively. The fear of introducing operational bias, impact of ADs on other important study objectives (such as safety and health economic evaluation) when trials are stopped early, and the potential of population drift as a result of the $A D$ and its implications on the interpretation of findings were viewed with varying degrees of concern among crosssector respondents. Supplementary summary data on cross-sector concerns about the use of ADs in confirmatory trials and detailed description of survey questions are provided (see Additional file 4).

\section{Organisational priority to the use of ADs in confirmatory trials}

When respondents were asked to rate the level of organisational priority they give to the use of ADs and/or research on ADs-related methods within the next 5 to 10 years, $15(50 \%)$ of the UK CTUs selected it as a 'medium priority' and just 3(10\%) as a 'high priority'. In contrast; $5(29 \%), 4(24 \%)$ and $4(24 \%)$ private organisations selected it as a 'medium priority', 'high priority', and an 'essential priority', respectively.

Only 2(7 \%) UK CTUs and 3(18\%) of private sector organisations reported having an AD-related Working Group within their organisation. The willingness to 'definitely consider' the use of ADs in future confirmatory trials, when appropriate, was expressed among 16(53\%) UK CTUs and 11(65\%) private sector organisations.

Forty-five $(55 \%)$ of the public funder respondents rated their boards or panels priorities on funding themes on confirmatory ADs in the next 5 to 10 years as at least a 'medium priority', 19(22\%) as 'high priority' and only $4(5 \%)$ as an 'essential priority'. As a funding board or panel, only $26(30 \%)$ reported that they had previously recommended funding a confirmatory $\mathrm{AD}$ grant proposal; however, $26(30 \%)$ did not respond to the question. When asked whether they would consider recommending a confirmatory $\mathrm{AD}$ grant proposal for funding in the future when appropriate to address research question(s); $42(49 \%)$ indicated that they "would definitely consider', $21(24 \%)$ 'might or might not consider', $1(1 \%)$ 'would not consider', and 22(26\%) did not respond to the question.

\section{Potential facilitators to the appropriate use of ADs in confirmatory trials}

We found consistency in respondents' perceptions across sectors regarding the usefulness of potential facilitators to enhance the appropriate use of ADs. Figure 6 summarises the respondents' opinions. The majority of respondents reported that the availability of a troubleshooting toolkit of specific questions which clinical trialists need to ask themselves when considering the different types of ADs would be 'somewhat useful': 23(92 \%) UK CTUs, 61(95 \%) public funders, and 12(92\%) private sector organisations. The overwhelming majority of respondents reported that the access to published case studies of implemented ADs focusing on aspects such as design and rationale, implementation, regulatory and statistical challenges; lessons learned; what went wrong; and facilitators to challenges would be 'very useful' to researchers. Twenty-three (92\%) CTUs and 57(89 \%) of the public funder respondents reported that the need for a consensus guidance document on the acceptable scope of ADs, which addressed issues tailored for publicly funded confirmatory trials, would be at least 'somewhat useful'.

The existence of a CONSORT statement tailored for ADs was selected as at least 'somewhat useful' to enhance transparency and completeness in the conduct and reporting of ADs by 56(88 \%) public funders, 23(92\%) CTUs, and 13(100 \%) private sector organisations. A total of $46(72 \%)$ of public funder respondents viewed that refresher training of funding boards and panel members to be familiar with AD-related issues could help them in the reviewing and commissioning process.

\section{Types of ADs implemented in confirmatory trials}

The submission of historical AD-related grant proposals for funding considerations was reported among 13(43\%) of the UK CTUs. Historical use of at least some type of $\mathrm{AD}$ in confirmatory trials was reported in $27 \%(8 / 30)$ of UK CTUs and $47 \%(8 / 17)$ of the private sector organisations that responded to the surveys. Table 1 describes the type and scope of ADs implemented in the public and private sector. The use of ADs appears not to be widespread across sector organisations; however, there are a small number of pacesetter organisations frequently using certain types of ADs as part of their research development programs. The most commonly used AD methods across the sector include sample size 


\begin{tabular}{|c|c|c|} 
CONSORT guidance tailored for ADs \\
Accessible publication of case studies
\end{tabular}

re-estimation (SSR), standard two-arm group sequential design (GSD), futility analyses using stochastic curtailment methods, and operational seamless $2 / 3$ design. Moreover, ADs with some form of futility stopping, such as dropping futile treatment arms or stopping trials, appeared popular, consistent with cross sector/disciplinary receptiveness.

\section{Discussion}

We need to emphasise that ADs are not appropriate for every trial. When contemplating the use of ADs, logistical as well as statistical considerations should be made on a trial-to-trial basis. These considerations include the accrual of the primary endpoint data in relation to the expected recruitment rate, the rationale for choosing the design, feasibility or practicalities of implementing the design in practice, and potential benefits against additional complexities in implementation. Some of the considerations are highlighted in our preceding research [12].

\section{Main findings}

Perceived barriers and concerns exist concerning the appropriate use of ADs in the confirmatory setting for key stakeholders in clinical trials research. Stakeholder perceptions about barriers are largely consistent across sectors, with a few exceptions that reflect differences in organisational funding structures, experiences, and the nature of study interventions. For example, the lack of bridge funding accessible to UK CTUs in the form of small grants to support design developmental work of time-consuming and complex ADs was reported as the major stumbling block to the routine use of ADs.

The most important cross-sector barriers appear to be connected to the dearth of practical implementation knowledge and experience, lack of applied training, and paucity of implemented case studies of ADs to facilitate practical learning and problem-solving. This is intertwined with the amount of time and effort required in the planning of ADs. Moreover, both the private sector and UK CTUs voiced concerns that they are under immense pressure to deliver on other competing priorities that are based on simpler traditional mainstream trial designs. Hence, they have limited time to support complex ADs, even when they are appropriate.

\section{Implications for practice}

There is cross-sector and multidisciplinary interest in the use of ADs when appropriate to answer research questions. The benefits of ADs can only be reaped when key barriers to their use are adequately addressed.

First, there seems to be a strong need on the part of public funders to address sources of funding accessible to UK CTUs wishing to support the use of relevant, complex, and time-consuming ADs. For instance, the MAMS design requires in-depth statistical simulations and time commitment. This developmental stage is often unfunded, with researchers taking risks betting on 
Table 1 Distribution of the type of adaptive designs (ADs) implemented in confirmatory trials and their frequency stratified by sector

\begin{tabular}{|c|c|c|c|c|c|c|}
\hline \multirow[t]{2}{*}{ Type of $A D$ and its description } & \multicolumn{3}{|c|}{ UK Clinical Trials Units (CTUs) } & \multicolumn{3}{|l|}{ Private sector } \\
\hline & $\begin{array}{l}\text { Number of } \\
\text { CTUs }\end{array}$ & $\begin{array}{l}\text { Number of } \\
\text { trials }\end{array}$ & $\begin{array}{l}\text { Missing } \\
\text { responses }\end{array}$ & $\begin{array}{l}\text { Number of } \\
\text { organisations }\end{array}$ & $\begin{array}{l}\text { Number of } \\
\text { trials }\end{array}$ & $\begin{array}{l}\text { Missing } \\
\text { responses }\end{array}$ \\
\hline Sample size re-estimation (SSR) & $7(23 \%)$ & & & $7(41 \%)$ & & \\
\hline Blinded SSR allowing for increase only & 4 & 4 & 1 & 2 & 11 & - \\
\hline Blinded SSR allowing for increase or decrease & 2 & 1 & 1 & 2 & 3 & - \\
\hline Unblinded SSR allowing for increase only & 2 & 5 & 1 & 2 & 10 & - \\
\hline Unblinded SSR allowing for increase or decrease & 2 & 5 & 1 & - & - & - \\
\hline Unblinded SSR based on promising zone concept & 2 & - & 2 & 3 & 10 & - \\
\hline Standard two-arm Group Sequential Design (GSD) & $7(23 \%)$ & & & $8(47 \%)$ & & \\
\hline Stopping early for futility only & 2 & 7 & 1 & 3 & 26 & - \\
\hline Stopping early for efficacy only & 1 & . & 1 & - & - & - \\
\hline Stopping early for efficacy or futility & 4 & 6 & - & 3 & 8 & 1 \\
\hline Stopping early for safety only & 4 & 2 & 2 & 2 & 5 & 1 \\
\hline Stopping early for safety or futility & 2 & 2 & - & 1 & 5 & - \\
\hline Stopping early for non-inferiority only & - & - & - & 1 & - & 1 \\
\hline Futility analysis (outside GSD framework) & $8(27 \%)$ & & & $5(29 \%)$ & & \\
\hline Based on conditional power & 5 & 7 & 1 & 3 & 3 & 2 \\
\hline Based on predictive power & 2 & 1 & 1 & 1 & - & 1 \\
\hline Based on confidence interval of the interim effect & 3 & 3 & 1 & - & - & - \\
\hline Operational seamless $2 / 3$ design & $7(23 \%)$ & & & $6(35 \%)$ & & \\
\hline Dropping futile treatment arms in phase 2 only & 5 & 5 & 1 & 3 & 3 & 1 \\
\hline Selecting only one promising treatment in phase 2 only & 1 & - & 1 & 3 & 2 & 1 \\
\hline Selecting multiple promising treatments in phase 2 only & - & - & - & 2 & 3 & 1 \\
\hline Other & 2 & - & 2 & - & - & - \\
\hline Inferential seamless $2 / 3$ design & $2(7 \%)$ & & & $3(18 \%)$ & & \\
\hline Dropping futile treatment arms in phase 2 only & 2 & 1 & 1 & 1 & 2 & - \\
\hline Addition or dropping futile treatment arms in phase 2 only & 1 & . & 1 & - & - & - \\
\hline Strictly phase 3 multi-arm multi-stage design & $2(7 \%)$ & & & $2(17 \%)$ & & \\
\hline Stopping trial for efficacy or futility or dropping futile treatment arms & 1 & 1 & - & - & - & - \\
\hline Information-based GSD & - & - & - & $4(24 \%)$ & 3 & 2 \\
\hline Standard GSD with SSR & - & - & - & $1(6 \%)$ & - & 1 \\
\hline Patient enrichment or subgroup selection & $2(7 \%)$ & & 2 & $2(12 \%)$ & - & 2 \\
\hline Response adaptive randomisation & $2(7 \%)$ & 2 & - & $2(12 \%)$ & 2 & 1 \\
\hline
\end{tabular}

Note: Denominator is based on responders; UK CTUs $(n=30)$ and private sector organisations $(n=17)$

uncertain future success of research grants. Hence, given the high risk involved, UK CTUs may be reluctant to support such ADs, even when they are more relevant to answer research questions efficiently. Although in the UK, the NIHR provides infrastructure funding accessible to accredited CTUs [21], this funding is often used for other purposes, such as meeting contractual obligations of staff who may not receive funding between studies. There is an opportunity for the NIHR and MRC to create a small funding stream to support the design of time-consuming designs provided the research questions meet their priority needs, and there is a strong design rationale. The funding should be conditional on open access publication of design-related material such as software programs to enhance planning of related future trials. We would also encourage the use of ADs, which are simple to implement within the existing scope of public funding models for fixed sample size designs, such as sample size reviews and futility assessments.

Most of the important barriers reported here are associated with a lack of practical knowledge and experience among key stakeholders. Numerous theoretical 
developments have occurred in ADs, and more are needed to address unknowns. However, what is lacking is a translational framework to enhance the use of the ADs in practice. We encourage accessible publication of case studies of successful and unsuccessful ADs with related materials. These publications should encompass aspects such as rationale and design; statistical and practical challenges, and how they are resolved; implementation resources; lessons learned; regulatory, data management and communication hurdles, and how these are resolved; and other facilitators to successful implementation. Learning from researchers or organisations who are routinely implementing ADs is paramount. Most importantly, a need exists for a focal group of practical experts who are publicly funded to support those CTUs with little practical expertise wishing to implement ADs. Furthermore, such experts should provide practical training accessible on ADs to UK CTUs. Although an initiative exists through the MRC Hubs for Trials Methodology Research Network AD Working Group [22], some trialists viewed it as being more theoretically oriented [12]. Most importantly, it seems that the time is upon us for close collaboration in the practical implementation of ADs.

We strongly encourage researchers who receive public funding for AD-related methodological research to produce open access resources such as free-to-use software or code to implement the methods developed. This would facilitate the application of the methods. A recommendation also is that CTUs receiving AD-related bridge or research funding should form a compendium of case studies for publication. Open-access publication of research outputs and resources such as in monographs is important. This could be an important resource aimed at reducing research waste and improving the appropriate conduct of adaptive trials. Such knowledge-sharing would be helpful for applied knowledge transfer.

Concerns regarding the robustness of ADs in decisionmaking and their credibility to change practice when trials are stopped early are real and should be addressed. Even though there are multi-dimensional aspects to these concerns, transparent and adequate reporting of trial conduct may alleviate some of the concerns. For instance, suboptimal reporting of appropriate statistical methods to obtain unbiased trial results (point estimates, $\mathrm{CIs}$ and P-values) following an $\mathrm{AD}$ may influence consumers of research findings to view them with suspicion [23-25]. There is a need for a CONSORT statement tailored for ADs to enhance their reporting and conduct. We support recent related initiatives proposing some adaptive trial aspects, which should be reported by researchers [26]. Case studies of ADs investigating a wide range of interventions published in 'high impact' journals and their influence on clinical practice could also help to convince sceptical research consumers.

Like any new methods, the use of ADs in confirmatory trials is bound to raise anxiety among some researchers. Some of this anxiety could be alleviated by a crossdisciplinary, consensus guidance document on ADs wellcrafted to addressing pertinent issues in confirmatory trials. For instance, the design of complex interventions has gone through a similar phase, but the emergence of related guidance documents improved researchers' receptiveness towards their conduct [27]. Most importantly, there is a need for a troubleshooting toolkit with pertinent design-specific questions, which trialists need to ask themselves when considering ADs. This could be helpful in the appropriate planning of ADs.

Optimal description of any proposed $\mathrm{AD}$ by researchers to key stakeholders (reviewers, funders/sponsors, collaborators and regulators) where appropriate is fundamental; its rationale within the context of the research objectives, potential benefits compared to mainstream traditional approach, scope, decision criteria to guide the adaptation and decision-making process, variable costs and trial durations, measures to minimise operational bias and control of statistical properties (type I error rate, power and inference), among others should be addressed. For instance, our preceding research [12] found that the fear of risking regulatory approval does not necessarily reflect regulatory perspective but is mostly an artefact of inadequate description of the proposed $\mathrm{AD}$ and its suitability to address the research question(s). There are encouraging indications that regulatory receptiveness to the appropriate use of ADs is positive, with improving awareness and experiences, particularly with respect to scientific advice and review of $\mathrm{AD}$ proposals [5, 12, 28, 29]. In this regard, as reflected in our preceding research [12], we encourage funders to modify their grant application forms to facilitate adequate description of $\mathrm{AD}$-related aspects. This could be achieved by allowing clinical trialists to add specific relevant $\mathrm{AD}$ material as appendices.

Periodic 'refresher training' of public funding boards and panel members prior to their commissioning meeting may help alleviate a lack of awareness of the acceptable scope of ADs, when they are appropriate, and their benefits in confirmatory trials. We believe the experience of funding boards and panels can only be improved when researchers put forward more appropriate ADrelated grant proposals for consideration. A positive change in attitudes and receptiveness towards appropriate use of ADs by public funders is an encouraging opportunity, which should be exploited by researchers.

The challenges faced by researchers in developing widely-acceptable decision-making criteria at the design 
stage to inform the adaptation process can be alleviated through multidisciplinary engagement and discussions during planning. This process should include close discussions among key stakeholders such as trial statisticians, clinicians, patient representatives, clinical peer advocate groups, and regulators. Our preceding study summarises some additional facilitators to the appropriate use of ADs [12].

\section{Interpretation of the findings}

This study is based on themes generated from crosssector and cross disciplinary in-depth interviews of key stakeholders involved in clinical trials research. Notably, the surveys explored the perceptions of funders and trialists towards ADs, predominantly in the UK confirmatory setting. Most importantly, this is the first study that employed Rating Scale Modelling to rank barriers in order of perceived importance to address the research question.

Some of our findings on barriers are consistent with the literature $[4,6,9,11]$. The most top-rated barriers, such as lack of practical knowledge, lack of applied training, time constraints to support planning, research teams preferring traditional mainstream designs to ADs, and limited access to case studies about ADs to facilitate practical training were reported in both the private and public sectors. In contrast, some barriers such as inadequate data management infrastructure to support ADs and associated practical complexities during planning were viewed by private sector organisations as more important than in the public sector. This could be partly explained by differences in experiences in the conduct of ADs - hence, a differential awareness of the practical challenges faced. This could also be due to the differences in the type of interventions under investigation and the regulatory framework.

It should be noted that our findings are based on moderate response rates across sectors. Most recent surveys of UK CTUs observed response rates of $38 \%$ [30] and ranged from $25 \%$ to $67 \%$ [31]. The private sector organisations we had contact with were invited to take part. The number of responders was similar to previous research by the $\mathrm{AD}$ Working Group (USA) although they achieved a $100 \%$ questionnaire response rate over a 1-year period [6]. To the best of our knowledge, we are not aware of previous research surveying public funders. Bearing all this in mind and based on email replies from non-responders (UK CTUs and public funders), the non-responders are likely different from the responders. For instance, non-responders seem more likely to be unfamiliar with ADs or do not view ADs as a priority. Hence, some of our findings on barriers, such as awareness of ADs, their benefits and acceptable scope in confirmatory setting, and lack of statistical expertise highlighted during interviews [12], could be underestimated. On the other hand, one could argue that such findings are conservative.

\section{Conclusions}

A general multi-disciplinary and cross-sector interest exists for the appropriate use of ADs. However, there are still persistent and important perceptions of organisational and individual roadblocks hampering the appropriate uptake of ADs in clinical trial practice. The lack of bridge funding accessible to UK CTUs wishing to support developmental design work seems to be an important obstacle requiring redress. A cross-sector collaboration and paradigm shift towards translational applied training, and accessible publication of case studies on ADs are paramount in addressing the dearth of knowledge and experience. Most importantly, a troubleshooting toolkit of key design-specific questions researchers need to ask themselves when considering ADs, transparent and adequate reporting of the conduct of adaptive trials, and a multidisciplinary consensus guidance document on the acceptable scope of ADs in confirmatory trials are all required to facilitate their appropriate use.

\section{Additional files}

Additional file 1: Supplementary summary data on UK Clinical Trials Unit (CTU) perceptions of important barriers to adaptive designs (ADs) use in confirmatory trials; Summary statistics. (PDF $88 \mathrm{~kb}$ )

Additional file 2: Supplementary summary data on public funder perceptions of important barriers to adaptive designs (ADs) use in confirmatory trials; Summary statistics. (PDF $75 \mathrm{~kb}$ )

Additional file 3: Supplementary summary data on private sector organisations' perceptions of important barriers to adaptive designs (ADs) use in confirmatory trials; Summary statistics. (PDF $157 \mathrm{~kb}$ )

Additional file 4: Cross-sector perceptions of concerns towards adaptive designs (ADs) use in confirmatory trials; Summary statistics. (PDF $33 \mathrm{~kb}$ )

\section{Abbreviations}

AD: adaptive design; Cl: confidence interval; CR UK: Cancer Research United Kingdom; CTU: Clinical Trials Unit; CRO: Contract Research Organisation; EMA: European Medicines Agency; EME: Efficacy Mechanism and Evaluation; FDA: Food and Drug Administration; GSD: group sequential design; HTA: Health Technology Assessment; NIHR: National Institute for Health Research; MHRA: Medicines and Healthcare products Regulatory Agency; MRC: Medical Research Council; NHS: National Health Service; R \& D: Research and Development; RfPB: Research for Patient Benefit; SSR: sample size re-estimation; UK CRC: United Kingdom Clinical Research Collaboration; MAMS: multi-arm multi-stage.

\section{Competing interest}

The authors declare that they have no competing interests.

\section{Authors' contributions}

All authors contributed to the design of the study and manuscript write-up. MD conducted the study, analysed the data, and led the write-up of the manuscript. ST, SJ and JN advised and helped MD as part of the NIHR DRF supervisory panel. JB advised MD in drawing up themes from in-depth 
interviews to construct survey instruments. All authors approved the final manuscript version.

\section{Acknowledgements}

The National Institute of Health Research fully funded MD as part of a Doctoral Research Fellowship (Grant Number: NIHR DRF-2012-05-182). SJ and JN are funded by the University of Sheffield. ST is funded by the University of Reading. JB is funded by the University of Hertfordshire. We express our gratitude to Mike Bradburn, Alicia O'Cathain, Cindy Cooper, and Daniel Hind for their useful comments and advice during this study as part of the fellowship advisory panel. We would also want to thank Tracey Young for her useful advice on Rasch Modelling. Finally, we are grateful for the generous and unwavering contribution of participating organisations and individuals to our understanding of the subject.

\section{Disclaimer}

The views expressed are those of the authors and not necessarily those of the National Health Service, the National Institute for Health Research, the Department of Health or organisations affiliated to or funding them.

\section{Author details}

${ }^{1}$ ScHARR, Regent Court, University of Sheffield, 30 Regent Street, S1 4DA, Sheffield, UK. 'Department of Mathematics and Statistics, University of Reading, Whiteknights, Reading RG6 6AX, UK. ${ }^{3}$ Centre for Research in Primary and Community Care, University of Hertfordshire, Hatfield AL109AB, Hertfordshire, UK.

Received: 3 July 2015 Accepted: 14 December 2015 Published online: 23 December 2015

\section{References}

1. Thompson WR. On the likelihood that One unknown probability exceeds another in view of the evidence of Two samples. Biometrika. 1933;25:285-94.

2. Bauer P, Bretz F, Dragalin V, König F, Wassmer G. Twenty-five years of confirmatory adaptive designs: opportunities and pitfalls. Stat Med. 2015 Mar 16. doi: 10.1002/sim.6472

3. Todd S. A 25-year review of sequential methodology in clinical studies. Stat Med. 2007;26:237-52.

4. Morgan CC, Huyck S, Jenkins M, Chen L, Bedding a, Coffey CS, et al. Adaptive design: results of 2012 survey on perception and Use. Ther Innov Regul Sci. 2014;48:473-81.

5. Elsäßer A, Regnstrom J, Vetter T, Koenig F, Hemmings RJ, Greco M, et al. Adaptive clinical trial designs for European marketing authorization: a survey of scientific advice letters from the European medicines agency. Trials. 2014;15:383.

6. Quinlan J, Gaydos B, Maca J, Krams M. Barriers and opportunities for implementation of adaptive designs in pharmaceutical product development. Clin Trials. 2010;7:167-73.

7. Chow S-C, Corey R. Benefits, challenges and obstacles of adaptive clinical trial designs. Orphanet J Rare Dis. 2011;6:79.

8. Chang M, Chow S-C, Pong A. Adaptive design in clinical research: issues, opportunities, and recommendations. J Biopharm Stat. 2006;16:299-309. discussion 311-2.

9. Kairalla J a, Coffey CS, Thomann M a, Muller KE. Adaptive trial designs: a review of barriers and opportunities. Trials. 2012;13:145.

10. Coffey CS, Levin B, Clark C, Timmerman C, Wittes J, Gilbert P, et al. Overview, hurdles, and future work in adaptive designs: perspectives from a National Institutes of Health-funded workshop. Clin Trials. 2012;9:671-80,

11. Jaki T. Uptake of novel statistical methods for early-phase clinical studies in the UK public sector. Clin Trials. 2013;10:344-6.

12. Dimairo M, Boote J, Julious SA, Nicholl JP, Todd S. Missing steps in a staircase: a qualitative study of the perspectives of key stakeholders on the use of adaptive designs in confirmatory trials. Trials. 2015;16:430

13. UKCRC Registered Clinical Trials Units Network. [http://www.ukcrc-ctu.org. uk/ Date accessed 14 November 2014.

14. NIHR. NIHR Annual Report 2013/14. NIHR; 2015. [http://www.nihr.ac.uk/ documents/about-NIHR/NIHR-Publications/NIHR-Annual-Reports/ NIHR\%20Annual\%20Report\%202013_2014.pdf]. Date accessed 1 June 2015.

15. Health Technology Assessment (HTA) Programme. [http://www.nets.nihr.ac. uk/programmes/hta]. Date accessed 20 August 2014
16. NIHR HTA Programme: Our people. [http://www.nets.nihr.ac.uk/ programmes/hta/our-people] Date accessed 20 August 2014.

17. Vagias, WM. (2006). Likert-type scale response anchors. Clemson International Institute for Tourism \& Research Development, Department of Parks, Recreation and Tourism Management. Clemson University. [https:// www.clemson.edu/centers-institutes/tourism/documents/sample-scales.pdf]. Date accessed 11 November 2013.

18. Andrich D. A rating formulation for ordered response categories. Psychometrika. 1978:43:561-73.

19. RUMM Laboratory Pty Ltd. RUMM2030. [http://www.rummlab.com.au/] Date accessed 20 August 2014.

20. Bennett C, Khangura S, Brehaut JC, Graham ID, Moher D, Potter BK, et al. Reporting guidelines for survey research: An analysis of published guidance and reporting practices. PLoS Med. 2011;8:1-11.

21. NIHR Clinical Trials Unit (CTU) Support Funding. [http://www.nets.nihr.ac.uk programmes/ctu] Date accessed 14 November 2014.

22. MRC Network of Hubs for Trials Methodology Research. [http://www. methodologyhubs.mrc.ac.uk/] Date accessed 14 November 2014.

23. Montori VM, Devereaux PJ, Adhikari NKJ, Burns KEA, Eggert CH, Briel M, et al. Randomized trials stopped early for benefit: a systematic review. JAMA. 2005;294:2203-9.

24. Wilcox RA, Djulbegovic B, Moffitt HL, Guyatt GH, Montori VM. Randomized trials in oncology stopped early for benefit. J Clin Oncol. 2008;26:18-9.

25. Bassler D, Montori VM, Briel M, Glasziou P, Guyatt G. Early stopping of randomized clinical trials for overt efficacy is problematic. J Clin Epidemiol. 2008;61:241-6.

26. Detry M, Lewis R, Broglio K, Connor J. Standards for the design, conduct, and evaluation of adaptive randomized clinical trials. 2012.[http://www. pcori.org/assets/Standards-for-the-Design-Conduct-and-Evaluation-ofAdaptive-Randomized-Clinical-Trials.pdf]. Date accessed 12 July 2014

27. Craig P, Dieppe P, Macintyre S, Michie S, Nazareth I, Petticrew M. Developing and evaluating complex interventions: the new Medical Research Council guidance. BMJ. 2008;337:a1655.

28. FDA. Adaptive designs for medical device clinical studies: draft guidance for industry and Food and Drug Administration staff. 2015.htttp://www.fda.gov/ ucm/groups/fdagov-public/@fdagov-meddev-gen/documents/document/ ucm446729.pdf]. Date accessed 20 May 2015.

29. Lin M, Lee S, Zhen B, Scott J, Horne A, Solomon G, Russek-Cohen E. CBER's experience with adaptive design clinical trials. Ther Innov Regul Sci. 2015: 2168479015604181. [http://dij.sagepub.com/content/early/2015/09/15/ 2168479015604181.abstract].

30. Bower P, Brueton V, Gamble C, Treweek S, Smith CT, Young B, et al. Interventions to improve recruitment and retention in clinical trials: a survey and workshop to assess current practice and future priorities. Trials. 2014;15:399

31. Tudur Smith C, Hickey H, Clarke M, Blazeby J, Williamson P. The trials methodological research agenda: results from a priority setting exercise. Trials. 2014;15:32.

\section{Submit your next manuscript to BioMed Central and we will help you at every step:}

- We accept pre-submission inquiries

- Our selector tool helps you to find the most relevant journal

- We provide round the clock customer support

- Convenient online submission

- Thorough peer review

- Inclusion in PubMed and all major indexing services

- Maximum visibility for your research

Submit your manuscript at www.biomedcentral.com/submit 